\title{
Access Pricing and Entry in the Postal Sector
}

\author{
FRANCIS BLOCH \\ GREQAM, Université de la Méditerranée and Warwick University \\ AXEL GAUTIER * \\ HEC, Université de Liège and CORE, Université Catholique de Louvain
}

\begin{abstract}
In a fully liberalized postal market, two business models will be possible for a new postal operator: (1) access: where the firm performs the upstream operations and uses the incumbent's network for final delivery and (2) bypass where the competing firm controls the entire supply chain and delivers mails with its own network. The choice between access and bypass depends on the entrant's delivery cost relative to the access price. In this paper, we derive welfare maximizing prices for the incumbent operator and we show how these prices should be re-balanced when the entry method is considered as endogenous.
\end{abstract}

\section{Introduction}

This paper concentrates on the ongoing liberalization process of the postal sector in the European Union. It focuses in the entry of new postal operators on the downstream segments of the postal market (the delivery of mails). Competition in the postal sector raises a major concern for the financing of the universal service obligations (USO) imposed on the incumbent operators. USO includes the requirement to serve all customers (universality/ubiquity), the imposition of a geographically uniform tariff for a bundle of products, obligations in term of service quality (frequency of delivery, accessibility of contact points), and constraints on prices. It is commonly accepted that universal service obligations are associated with large fixed costs for the universal service provider (USP). ${ }^{1}$

For the moment, the universal service obligations are (partially) financed by monopoly profits in the reserved areas. As these reserved areas will disappear, so will the associated monopoly profits (or at least part of them). In this case, the future of USO is no longer guaranteed and the financing of the USO becomes a major concern for both the regulator

\footnotetext{
* Contact author. Axel Gautier, HEC-ULg, Bat B31, Boulevard du rectorat, 7, B4000 Liège, Belgium. Email: agautier@ulg.ac.be. Axel Gautier is the winner of the Post-Doctoral Grant in Postal Economics offered by the Belgian Post. He gratefully acknowledges the financial support from the sponsor. The view expressed here does not necessarily reflect those of the Belgian Post. The authors appreciated the comments and the remarks from participants at the Fourth Conference on "Regulation, Competition and Universal Service in the Postal Sector" held at IDEI (Toulouse) in March 2006 and from an anonymous referee.

${ }^{1}$ Cazals et al (1997), Cremer et al (1997).
} 
(or the State) and the USP. The extent of this problem will obviously depend on the scope of the USO and the importance of competition in the postal markets.

Maintaining the USO in a competitive market is thus a source of tension. To overcome this potential problem, proposals have been made to limit the scope of the USO and/or to limit competition to the upstream segments of the market. ${ }^{2}$ Moreover, the welfare impact of entry of competitors on the postal markets is not clear-cut. For example, Crew and Kleindorfer (2006) provide examples where the impact on welfare of allowing downstream bypass is negative. Panzar (2005) shows that the development of both upstream and downstream competition is not in conflict with the pursuit of public policies (low prices, USO coverage) as long as piecemeal bypass (the possibility for mailers to buy access from a competitor) is not allowed. In his model, the development of E2E mail competition reduces the incumbent's mail volume and therefore, the incumbent should charge a higher margin on its products to cover the fixed costs.

This paper concentrates on the impact of the incumbent's pricing behavior on the entry of the competitors on the postal market. A potential competitor of the incumbent postal operator has different entry strategies: it can either deliver its mails using the existing delivery network of the incumbent operator (downstream access) or it can deliver its mails using its own delivery network (downstream bypass). If the competitor chooses downstream access, it performs only the upstream operations (collection, sorting, transport) and uses the incumbent's delivery network for which it pays an access price. If the competitor chooses downstream bypass, it performs all the upstream and downstream operations on its own. ${ }^{3}$

In Sweden, CityMail chose the downstream bypass option and delivers mails with its own delivery network. However, CityMail has a limited geographical coverage. Sandd in the Netherlands has now a nationwide delivery network and achieves a market share of 8\%. In the UK, UK-Mail (and many others) offers E2E mail services but the mails collected by UK-Mails are ultimately delivered by the incumbent operator, Royal Mail (downstream access).

This paper builds on the literature on efficient access pricing in the postal sector (Crew and Kleindorfer, 1992; De Donder, 2006; Billette de Villemeur et al 2007; and Laffont and Tirole, 1994, 2000). In the efficient access pricing approach, the incumbent's stamp and access prices are derived by maximizing the total welfare while guaranteeing a nonnegative profit for the firms. If USO obligations are imposed (or if there are fixed costs in the delivery activity), the access price paid by the entrant to the incumbent operator is equal to the incumbent's marginal cost of delivery plus a mark-up. This mark-up aims at covering part of the fixed costs associated with the incumbent's USO. It can be decomposed into a "Ramsey term" and a "displacement term". For each product, including access, the Ramsey term is inversely related to the product's price elasticity. Products for which the demand is highly sensitive to prices are charged a lower mark-up than those who are relatively less price sensitive. The displacement term is the product of the incumbent's margin on its E2E products and the displacement ratio which measures the substitutability between the incumbent's and the entrant's products. If the entrant attracts a large fraction of the incumbent's customers (the displacement ratio is high), competition creates serious concerns for the USO financing. Therefore, the regulator sets a high access charge to levy

\footnotetext{
${ }^{2}$ As in the US situation where the incumbent firm USPS maintains a monopoly position on the last mail delivery.

${ }^{3}$ Unlike Panzar (2005), we do not consider competitors that perform only downstream operations.
} 
a large contribution to the USO financing from the entrant. Conversely, if the entrant offers innovative products, and attract few consumers from the incumbent but rather new consumers, its contribution to the USO financing will be lower. ${ }^{4}$

This paper is closely related to the work by Billette de Villemeur et al (2007) on optimal pricing under bypass and access. Billette de Villemeur et al (2007) consider a more general and more detailed demand structure (allowing for two types of customers: E2E mail and workshared mail), and compute the optimal pricing rules under bypass and access. Our computation of optimal prices is directly inspired by their work. We view our paper as complementary to theirs: we provide a full welfare analysis of the choice of delivery method, and analyze in detail the effect of incumbent's prices on the entry choice of the competitor, two aspects which are absent in Billette de Villemeur et al (2007), but turn out to be extremely important in practice.

\subsection{Overview of the results}

In this paper, we start by deriving the efficient access and stamp prices in the case where the entrant buys access to the incumbent's delivery network, and in the case in which it bypasses and builds up its own delivery network. We start our analysis by considering that the incumbent's stamp prices depend on the delivery region (non-uniform tariff). In the case of downstream access, the access price is the sum of delivery cost, a Ramsey term and a displacement term. Under bypass, there are two modifications in prices: (a) the tariff is rebalanced and the incumbent is relatively more aggressive in the urban market where it faces competition, and (b) because the USP loses access receipts, there is an overall increase of all its prices to cover the fixed costs associated with the USO. These results corroborate those of Billette de Villemeur et al (2007).

Next, we compute the efficient delivery choice for the entrant. One needs to distinguish between the first best delivery method (which maximizes welfare) and the second best method (which minimizes delivery cost). On the basis of costs, the entrant should by-pass the incumbent as long as its marginal delivery cost is lower than the marginal delivery cost of the incumbent. However, taking into account the USO and the fact that the incumbent needs to cover the fixed cost of maintaining his delivery network, the first best delivery method involves access even when the entrant has a lower delivery cost. This result shows that an entrant who chooses his delivery method on the basis of costs will bypass for a range of parameters for which access is socially efficient. Hence, excess bypass can arise on the market, but excess access never occurs. Furthermore, it is interesting to note that bypass may not be the efficient delivery method when the delivery cost of the entrant is too low. In that case, competition may drive the profits of the incumbent below the cost of USO, hence lead the regulator to prefer access.

We then consider the problem faced by a regulator who cannot choose the delivery method of the entrant, and must take into account the entrant's incentive as a new constraint to his maximization problem. We then show that the regulator's choice will be aligned with delivery cost minimization: the regulator promotes access when the incumbent has a lower delivery cost, and bypass when the entrant has a lower delivery cost.

\footnotetext{
${ }^{4}$ Displacement ratios are likely to be high in the postal sector. For example De Donder et al (2006) calibrate a model with displacement ratios of 0.75 and 0.9 , meaning that three quarter (or 9 over 10) of the mails treated by the competitors are displaced from the incumbent.
} 
When a uniform tariff is imposed on the USP, the incumbent postal operator has less freedom to set prices and it therefore has an impact on the entry strategy of the competitor. As urban mail price increases, the entrant is able to capture a larger fraction of the urban mail demand with a given price. In other words, the displacement ratio is higher. As a consequence, the efficient access price increases which makes bypass more attractive for the entrant. Then, inducing the efficient choice of access requires more distortion in the prices than in the case of non-uniform tariff. This means that the cost in terms of welfare of having an efficient delivery method is higher.

We also consider a situation of incomplete information, when the regulator and the USP only know the prior distribution of the entrant's delivery costs but not its realization. This uncertainty in costs translates into an uncertainty in the choice of a delivery method. The probability of entry with bypass is endogenous and it depends on the access and stamp prices. ${ }^{5}$ The regulator then faces a trade-off between promoting efficiency, that is, reducing the probability of bypass, which requires a low access price and USO financing which instead requires a high access price. We show that uncertainty on the entrant's delivery cost could induce the entrant to bypass with a positive probability, even if it is not ex-post efficient.

Our approach differs from the Efficient Component Pricing Rule (ECPR) which also takes the market structure as endogenous. In the ECPR approach (Armstrong, 2001, for example), it is efficient to have one producer serving the entire market segment. The ECPR pricing rule is then designed to select the most efficient producer. By setting the access price equal to the incumbent's opportunity cost, that is, its retail price minus its cost, the ECPR gives the right incentive to the entrants. They enter the market only if they are more efficient than the incumbent operator. In our approach, the prices are not designed to select the most efficient producer but to maximize the welfare taking into account that the regulator does not control the choice of the delivery method by the entrant. ${ }^{6}$

The contribution of this paper is paper to analyze the impact of pricing on the entry strategy of the competitor, that is, how prices should be adapted to take into account their impact on the entry behavior of the competitors. By doing so, we neglect other issues like the reform of the USO. Clearly, relaxing some of the USO constraints is another possible way to overcome the problem of USO financing in a competitive market (see Crew and Kleindorfer, 2006).

\section{Model}

There are two postal operators on the market: an incumbent postal operator - we call it firm 1 - and an entrant - firm 2. By assumption, USO are imposed on firm 1 only and the regulatory regime is an asymmetric one: firm 1 is fully regulated while firm 2 is not. However, we will assume that firm 2 behaves competitively and prices mails at marginal cost.

\footnotetext{
${ }^{5}$ This part of the paper is closely related to Dam et al (2007) who develop Ramsey pricing formulas that apply when the entry on the market is endogenous.

${ }^{6}$ ECPR and efficient prices will only coincide in exceptional circumstances. See for example Laffont and Tirole (2000), Armstrong, Doyle and Vickers (1996).
} 
Mails are delivered in two delivery zones: a high density region (urban) and a low density region (rural). Delivery costs depend on the population density and differ in the two regions.

Each postal operator offers end-to-end (E2E) mails to consumers. Firm 1 delivers mail to both regions. This ubiquity constraint is part of the USO imposed on the incumbent. Firm 2 serves only the most profitable, urban, region. In the sequel, we use indices $i=1,2$ to refer to firms and exponents $k=u, r$ refer to delivery zones. The mail products of the two firms are differentiated. For example, the incumbent operator offers J+1 E2E mails to the customers while the entrant offers mail services with a lower frequency of delivery. ${ }^{7}$ Customers view the two products as imperfect substitutes. Moreover, we consider that the degree of product differentiation is not affected by the method chosen for delivery.

There are two types of costs associated with the production of E2E mails: an upstream and a downstream (delivery) cost. We represent by $c_{i}$ the unit cost of all the upstream operations (collection, sorting, transport, ...) for firm $i=1,2$. This upstream cost is independent of the delivery region. There is a constant unit cost of $d_{1}^{k}$ per mail delivered in region $k$ by firm 1 . Delivery costs are higher in the rural region: $d_{1}^{r}>d_{1}^{u}$. For the entrant, the unit cost of delivering one mail in the urban region is $d_{2}^{u}$. The entrant can avoid this delivery cost by buying access to the delivery network of firm 1 . In this case, it pays a per-unit access charge denoted by $\alpha^{u}$ to firm 1 and firm 1 supports the delivery cost $d_{1}^{u}$. In addition to these costs, the USO results in a fixed cost for the USP denoted by $F$.

The net surplus of a representative consumer who sends $x_{1}^{k}$ mails to zone $k$ with the incumbent and $x_{2}^{u}$ mails to the urban zone with the entrant is:

$$
U\left(x_{1}^{u}, x_{1}^{r}, x_{2}^{u}\right)-p_{1}^{u} x_{1}^{u}-p_{1}^{r} x_{1}^{r}-p_{2}^{u} x_{2}^{u}
$$

where the $p_{i}^{k}$ are the prices charged by firm $i$ for their E2E mails delivered in zone $k$.

The maximization of this surplus function gives the demand for each type of mail $x_{i}^{k}$. We will make the following assumptions on the demand functions:

1. $\frac{\partial x_{1}^{u}}{\partial p_{1}^{r}} \leq 0$ and $\frac{\partial x_{1}^{r}}{\partial p_{1}^{u}} \leq 0$

2. $\frac{\partial x_{2}^{u}}{\partial p_{1}^{r}}=0$ and $\frac{\partial x_{1}^{r}}{\partial p_{2}^{u}}=0$

Part 1 means that consumers give a positive value to the ubiquity of the service offered by the USP. Urban and rural mails offered by firm 1 are thus complements. In addition, the

\footnotetext{
${ }^{7}$ Sandd and CityMail deliver letters twice a week. Most of the mail providers that choose access offer a day definite mail delivery, 2 or 3 days after the mail collection.
} 
demand functions satisfy the standard following properties: $\frac{\partial x_{i}^{k}}{\partial p_{i}^{k}}<0, \frac{\partial x_{i}^{u}}{\partial p_{j}^{u}}>0$ and $\left|\frac{\partial x_{i}^{u}}{\partial p_{j}^{u}}\right| \geq \frac{\partial x_{i}^{u}}{\partial p_{j}^{u}}$.

In the sequel, we use elasticities in the pricing formulas. These elasticities are defined as follows:

\section{DEFINITIONS:}

1. The direct price elasticity (in absolute value) of a product sold by firm $i$ in region $k$ is:

$\eta_{i}^{k}=-\frac{\partial x_{i}^{k}}{\partial p_{i}^{k}} \frac{p_{i}^{k}}{x_{i}^{k}}$

2. The cross price elasticity of a product sold by firm $i$ on the urban market is, for $i, j=1,2$ :

$\eta_{i}^{u u}=\frac{\partial x_{i}^{u}}{\partial p_{j}^{u}} \frac{p_{j}^{u}}{x_{i}^{u}}$

3. The intra-brand price elasticities for firm 1 are: $\eta_{1}^{u r}=\frac{\partial x_{1}^{u}}{\partial p_{1}^{r}} \frac{p_{1}^{r}}{x_{1}^{u}}$ and $\eta_{1}^{r u}=\frac{\partial x_{1}^{r}}{\partial p_{1}^{u}} \frac{p_{1}^{u}}{x_{1}^{r}}$.

Consistent with our assumptions, we have positive cross price elasticities and negative intra-brand price elasticities.

The total welfare is the sum of the consumer's and producers' surplus:

$$
W=U\left(x_{1}^{u}, x_{1}^{r}, x_{2}^{u}\right)-\left(c_{1}+d_{1}^{u}\right) x_{1}^{u}-\left(c_{1}+d_{1}^{r}\right) x_{1}^{r}-\left(c_{2}+\theta d_{1}^{u}+(1-\theta) d_{2}^{u}\right) x_{2}^{u}-F
$$

where $\theta$ is a dummy variable that has a value of one if firm 2 chooses to buy access to deliver its mails and equals zero if it chooses bypass. The aim of the regulator is to set the stamp prices $p_{1}^{u}, p_{1}^{r}$ and the access price $\alpha^{u}$ in order to maximize the total welfare $W$. We will consider sequentially two cases. In the first one, the stamp price for mails to the urban and the rural area can be different. In the second one, a geographically uniform tariff is imposed: $p_{1}^{u}=p_{1}=p_{1}^{r}$.

Firm 2 behaves as a competitive fringe. The competitive fringe assumption means that its product is sold at marginal cost. Since this cost depends on the delivery method, the stamp price charged by firm 2 is for its urban mail is:

$$
\begin{aligned}
& p_{2}^{u}=c_{2}+\alpha^{u} \text { if firm } 2 \text { chooses access, } \\
& p_{2}^{u}=c_{2}+d_{2}^{u} \text { if firm } 2 \text { chooses bypass. }
\end{aligned}
$$


With this marginal cost pricing rule, the profit of firm 2 is always equal to zero. We assume that firm 2 chooses the delivery technology with the lowest cost, that is access if $\alpha^{u} \leq d_{2}^{u}$ and bypass otherwise. ${ }^{8}$

\section{$3 \quad$ Non-uniform pricing.}

In this section, we derive the welfare maximizing prices when the regulator can set different stamp prices for mails delivered in the urban and the rural regions. For convenience, the technical analysis is relegated to the appendix.

\subsection{Access}

Suppose that firm 2 uses firm 1's delivery network for which it pays an access fee of $\alpha^{u}$ per mail distributed. If firm 2 chooses access, the welfare is:

$$
W^{a}=U\left(x_{1}^{u}, x_{1}^{r}, x_{2}^{u}\right)-\left(c_{1}+d_{1}^{u}\right) x_{1}^{u}-\left(c_{1}+d_{1}^{r}\right) x_{1}^{r}-\left(c_{2}+d_{1}^{u}\right) x_{2}^{u}-F
$$

The regulator selects the prices that maximize welfare and that guarantee to firm 1 a non-negative profit that is, firm 1 is able to cover its costs (including the fixed cost of the USO) with its receipts from E2E mails and from access. This zero profit constraint is:

(1) $\Pi_{1}^{a}=\left(p_{1}^{u}-c_{1}+d_{1}^{u}\right) x_{1}^{u}+\left(p_{1}^{r}-c_{1}+d_{1}^{r}\right) x_{1}^{r}+\left(\alpha^{u}-d_{1}^{u}\right) x_{2}^{u}-F \geq 0$

Firm 2 sells its mail product at marginal cost and charges a price equal to:

(2) $p_{2}^{u}=c_{2}+\alpha^{u} \Rightarrow \Pi_{2}=0$

The regulator's objective is to find the stamp prices for the incumbent and the access price that maximize the welfare subject to the constraint (1). We denote by $\lambda$ the Lagrange multiplier of the zero profit constraint for firm 1. (2) implies that the derivative of the demand $x_{i}^{k}$ with respect to $\alpha^{u}$ is equal to the derivative of $x_{i}^{k}$ with respect to $p_{2}^{u}$. The first-order conditions to this problem read as follows:

$$
\begin{aligned}
& (1+\lambda)\left(\left(p_{1}^{u}-c_{1}-d_{1}^{u}\right) \frac{\partial x_{1}^{u}}{\partial p_{1}^{u}}+\left(p_{1}^{r}-c_{1}-d_{1}^{r}\right) \frac{\partial x_{1}^{r}}{\partial p_{1}^{u}}+\left(\alpha^{u}-d_{1}^{u}\right) \frac{\partial x_{2}^{u}}{\partial p_{1}^{u}}\right)+\lambda x_{1}^{u}=0 \\
& (1+\lambda)\left(\left(p_{1}^{u}-c_{1}-d_{1}^{u}\right) \frac{\partial x_{1}^{u}}{\partial p_{1}^{r}}+\left(p_{1}^{r}-c_{1}-d_{1}^{r}\right) \frac{\partial x_{1}^{r}}{\partial p_{1}^{r}}\right)+\lambda x_{1}^{r}=0
\end{aligned}
$$

\footnotetext{
${ }^{8}$ This assumption is identical to the assumption on the behavior of the competitive fringe in contestable markets (Baumol, Panzar and Willig, 1982).
} 
(5) $\quad(1+\lambda)\left(\left(p_{1}^{u}-c_{1}-d_{1}^{u}\right) \frac{\partial x_{1}^{u}}{\partial p_{2}^{u}}+\left(\alpha^{u}-d_{1}^{u}\right) \frac{\partial x_{2}^{u}}{\partial p_{2}^{u}}\right)+\lambda x_{2}^{u}=0$

This type of first-order conditions is standard in efficient access pricing problems (see, for example, De Donder, 2006). Rearranging the terms in the first-order conditions, we can express the prices as the sum of three terms: price $=$ marginal cost + a Ramsey term $+\mathrm{a}$ displacement term. That is:

(6) $\quad \alpha^{u}=d_{1}^{u}+\frac{\lambda}{1+\lambda} \frac{p_{2}^{u}}{\eta_{2}^{u}}+\left(p_{1}^{u}-c_{1}-d_{1}^{u}\right) \sigma^{u}$

where $\eta_{2}^{u}$ is the price elasticity of urban mail of firm 2 (in absolute value) and $\sigma^{u} \in[0,1]$ is the displacement ratio: $\sigma^{u}=\frac{d x_{1}^{u} / d p_{2}^{u}}{d x_{2}^{u} / d p_{2}^{u}}$.

Similarly, the stamp prices for the incumbent's mails are:

(7) $p_{1}^{u}=c_{1}+d_{1}^{u}+\frac{\lambda}{1+\lambda} \frac{p_{1}^{u}}{\eta_{1}^{u}}+\left(\alpha^{u}-d_{1}^{u}\right) \tilde{\sigma}^{u}+\left(p_{1}^{r}-c_{1}-d_{1}^{r}\right) \sigma^{u r}$

(8) $\quad p_{1}^{r}=c_{1}+d_{1}^{r}+\frac{\lambda}{1+\lambda} \frac{p_{1}^{r}}{\eta_{1}^{r}}+\left(p_{1}^{u}-c_{1}-d_{1}^{u}\right) \sigma^{r u}$

where $\eta_{1}^{k}$ is the price elasticity of mails sent to region $k$ by firm 1 and $\tilde{\sigma}^{u} \in[0,1]$ and $\tilde{\sigma}^{u}=-\frac{d x_{2}^{u} / d p_{1}^{u}}{d x_{1}^{u} / d p_{1}^{u}} ; \sigma^{u r} \in[-1,0]$ and $\sigma^{u r}=-\frac{d x_{1}^{r} / d p_{1}^{u}}{d x_{1}^{u} / d p_{1}^{u}} ; \sigma^{r u} \in[-1,0]$ and $\sigma^{r u}=-\frac{d x_{1}^{u} / d p_{1}^{r}}{d x_{1}^{r} / d p_{1}^{r}}$.

Solving this system, the efficient prices can be expressed as:

(9) $\quad M_{1}^{u}=\frac{p_{1}^{u}-c_{1}-d_{1}^{u}}{p_{1}^{u}}=\frac{\lambda}{1+\lambda}\left(\frac{1}{\hat{\eta}_{1}^{u}}+\gamma_{1}^{u}\right)$,

where $\hat{\eta}_{1}^{u}=\frac{\eta_{1}^{r} \eta_{1}^{u}-\eta_{1}^{r u} \eta_{1}^{u r}}{\eta_{1}^{r}+\eta_{1}^{r u}}$ and $\gamma_{1}^{u}>0$. Similarly,

(10) $M_{1}^{r}=\frac{p_{1}^{r}-c_{1}-d_{1}^{r}}{p_{1}^{r}}=\frac{\lambda}{1+\lambda}\left(\frac{1}{\hat{\eta}_{1}^{r}}+\gamma_{1}^{r}\right)$, 
where $\hat{\eta}_{1}^{r}=\frac{\eta_{1}^{r} \eta_{1}^{u}-\eta_{1}^{r u} \eta_{1}^{u r}}{\eta_{1}^{u}+\eta_{1}^{u r}}$ and $\gamma_{1}^{r}<0$

To start the discussion of these optimal prices, it is convenient to recall that, in the absence of competition, the optimal stamp price in region $k$ would be set at a level such that $\frac{p_{1}^{k}-c_{1}-d_{1}^{k}}{p_{1}^{k}}=\frac{\lambda}{1+\lambda} \frac{1}{\hat{\eta}_{1}^{k}}$. In other words, the optimal prices for a monopolist are inversely related to the so-called super-elasticities of its products. ${ }^{9}$ Because urban and rural mails are complements, we have $\hat{\eta}_{1}^{k}>\eta_{1}^{k}$ for $k=u, r$. How are prices modified with the presence of a competitor buying access? There are two main modifications. First, if entry is not neutral with respect to the incumbent's profit, that is, if the access receipts less than compensate the lost receipts from the customers, there is an overall increase in prices. This change is captured by an increase in the value of the Lagrange multiplier $\lambda$. Second, the tariff is rebalanced. This change is captured by the " $\gamma$ " terms in the above expressions. This " $\gamma$ " term is positive in the urban market and negative in the rural market. The displacement ratios explain these modifications in the prices. ${ }^{10}$ In the sequel, we denote by $p_{1}^{k a}, k=u, r$ and $\alpha^{*}$, the optimal prices that the regulator applies if the entrant buys access.

\subsection{Bypass}

Now suppose that firm 2 bypasses the incumbent's delivery network. The regulator selects the prices $p_{1}^{k}$ in order to maximize:

$$
W^{b}=U\left(x_{1}^{u}, x_{1}^{r}, x_{2}^{u}\right)-\left(c_{1}+d_{1}^{u}\right) x_{1}^{u}-\left(c_{1}+d_{1}^{r}\right) x_{1}^{r}-\left(c_{2}+d_{2}^{u}\right) x_{2}^{u}-F
$$

subject to:

(11) $\Pi_{1}^{b}=\left(p_{1}^{u}-c_{1}+d_{1}^{u}\right) x_{1}^{u}+\left(p_{1}^{r}-c_{1}+d_{1}^{r}\right) x_{1}^{r}-F \geq 0$

Firm 2 sells its mail product at marginal cost which leads to:

$$
p_{2}^{u}=c_{2}+d_{2}^{u} \Rightarrow \Pi_{2}=0
$$

In the case of bypass, there is (by definition) no access receipts and therefore the value of $\alpha^{u}$ is not meaningful in this case. The first order conditions can be expressed as:

$$
p_{1}^{u}=c_{1}+d_{1}^{u}+\frac{\lambda}{1+\lambda} \frac{p_{1}^{u}}{\eta_{1}^{u}}+\left(p_{1}^{r}-c_{1}-d_{1}^{r}\right) \sigma^{u r}
$$

\footnotetext{
${ }^{9}$ Super-elasticities were introduced by Rohlfs (1979). Their importance for access pricing in network industries has been emphasized by Laffont and Tirole (1994, 2000).

${ }^{10}$ See Billette de Villemeur et al (2007) for a related discussion.
} 
(14) $p_{1}^{r}=c_{1}+d_{1}^{r}+\frac{\lambda}{1+\lambda} \frac{p_{1}^{r}}{\eta_{1}^{r}}+\left(p_{1}^{u}-c_{1}-d_{1}^{u}\right) \sigma^{r u}$

Given that the incumbent does not collect any access revenues, it must raise its stamp prices in order to satisfy the zero-profit condition. Hence, at least one of the optimal stamp prices must be higher under bypass than under access. Furthermore, the displacement term disappears in equation (13) defining the optimal urban stamp price. This suggests that the relative price of urban mail with respect to rural mail is lower under bypass than under access, reflecting increased competition on the urban market.

Solving the system, the prices can be inversely related to the superelasticity of the products:

(15) $M_{1}^{k}=\frac{p_{1}^{k}-c_{1}-d_{1}^{k}}{p_{1}^{k}}=\frac{\lambda}{1+\lambda} \frac{1}{\hat{\eta}_{1}^{k}}$

Under bypass, the price structure is similar to that of monopoly prices but the overall level of prices is higher since the firm faces a stronger financial constraint. The firm needs to raise its prices to compensate for the lost receipts on the urban market due to the presence of the competitor. Under bypass, it is possible that the incumbent does not manage to break even. If the entrant's price is low enough and it captures large mail volumes on the urban market, the incumbent may find it impossible to break even, and a graveyard spiral takes place. ${ }^{11}$ In this case, it may become impossible to sustain the USO. In the sequel, we denote the prices under bypass by $p_{1}^{k b}, k=u, r$.

\subsection{Access vs. bypass}

We now compare welfare under bypass and access, $W^{a}$ and $W^{b}$ when the regulator controls the delivery decision of the entrant. The regulator can effectively exercise this control by putting restrictions on the license granted to the entrant. We establish that:

Proposition 1: There exists a cut-off point $d^{*}<d_{1}^{u}$ such that $W^{a} \geq W^{b}$ for all $d_{2}^{u} \geq d^{*}$.

Proof: We first show that $W^{a} \geq W^{b}$ for any $d_{2}^{u} \geq d_{1}^{u}$ (with strict inequality if $\left.d_{2}^{u}>d_{1}^{u}\right)$. The argument is a revealed-preference argument. In a model with access, the regulator could replicate the bypass prices by setting $\alpha^{u}=d_{2}^{u}, p_{1}^{u a}=p_{1}^{u b}, p_{1}^{r a}=p_{1}^{r b}$. This would result in a welfare level $\bar{W}^{a}$. As long as $d_{2}^{u} \geq d_{1}^{u}, \bar{W}^{a} \geq W^{b}$. Furthermore, as $\alpha \geq d_{1}^{u}, \bar{\Pi}_{1}^{a}=\Pi_{1}^{b}+\left(\alpha^{u}-d_{1}^{u}\right) x_{2}^{u} \geq \Pi_{1}^{b}=0$. So the choice $\alpha^{u}=d_{2}^{u}, p_{1}^{u a}=p_{1}^{u b}, p_{1}^{r a}=p_{1}^{r b}$ is a feasible choice for the regulator. Hence $W^{a} \geq \bar{W}^{a} \geq W^{b}$.

Suppose $d_{2}^{u}=d_{1}^{u}$. We can still show that $W^{a} \geq W^{b}$. The argument is that the optimal choice of $\alpha^{u}$ must involve $\alpha^{*} \geq d_{1}^{u}$ (see the equation characterizing the optimal choice of

\footnotetext{
${ }^{11}$ See Crew and Kleindorfer (2005).
} 
$\alpha$ ). Intuitively, the regulator prefers that the incumbent make a positive profit on the market where prices are not distorted, rather than raise prices to make sure that the profit constraint is satisfied. As $W^{a}$ and $W^{b}$ are continuous functions of $d_{2}^{u}$ and $d_{1}^{u}$, this shows that there exists $\varepsilon>0$ such that $W^{a} \geq W^{b}$ for all $d_{1}^{u} \geq d_{2}^{u}-\varepsilon$.

This proposition shows that as long as the entrant's marginal cost of delivery is higher than the incumbent's marginal cost of delivery in the urban area, access is the efficient (welfare maximizing) delivery method. If the two firms have identical delivery costs, the regulator still prefers access to bypass, since it raises money to finance the USO with access but not with bypass. By continuity of the welfare functions, this must also hold for $d_{1}^{u} \geq d_{2}^{u}-\varepsilon$. Hence, we observe that there exists a range of delivery costs for the entrant where the delivery cost of the entrant is smaller than that of the incumbent $\left(d_{2}^{u}<d_{1}^{u}\right)$, but the regulator would prefer that the entrant buy access from the technologically inefficient operator.

Some remarks are in order. First, a key assumption for proposition 1 is that the quality of the entrant's product is independent of the delivery technology. The two firms sell different products but the entrant sells the same product using the two delivery methods. In other words, it is assumed that the entrant cannot increase its product quality by controlling the whole supply chain. Of course, if it was not the case, the welfare under bypass would be higher and the parameter space under which bypass is efficient would increase. Second, we take for granted that the USO is financed by the USP's profits and the access receipts. We neglect other financing methods like the compensation fund (a method that is explicitly provided in French postal law but not yet used). ${ }^{12}$ Third, we implicitly assume that upstream competition is welfare enhancing. This is in fact the case if the entrant's product is sufficiently differentiated and/or the upstream $\cos c_{2}$ is not too great compared to $c_{1}$.

Notice that Proposition 1 is silent on the relation between the welfare obtained under access and bypass when the delivery cost of the entrant is below $d^{*}$. Intuitively, one would expect that bypass is preferred to access when the technological advantage of the entrant (measured by the difference $d_{1}^{u}-d_{2}^{u}$ ) is high. This is not necessarily the case, because bypass would then induce a very low price $p_{2}^{u}$, hence a high level of competition in the urban market which may prevent the operator from recovering its cost. Hence, the regulator may prefer to force access even though delivery costs of the entrant are very low, in order to guarantee nonnegative profits to the USP. We illustrate this with the following example:

EXAMPLE 1: Suppose that there only two urban markets, with utility $U\left(x_{1}, x_{2}\right)=x_{1}+x_{2}-\frac{1}{2}\left(x_{1}\right)^{2}-\frac{1}{2}\left(x_{2}\right)^{2}-\beta x_{1} x_{2}$. Let the upstream costs be given by $c_{1}=$ $c_{2}=0$, the downstream costs be given by $d_{1}$ and $d_{2}$.

Under access, it is easy to see that the optimal prices are given by

\footnotetext{
${ }^{12}$ Finland and Italy both rely on a compensation fund to partially finance the USO in a competitive market. However, in Finland, the level of the compensation fund tax has been so high as to prevent the entry of competitors. In Italy, hardly any taxes have been collected under the compensation fund even though they are legally required. (See the recent report by Price Waterhouse Coopers (2006) on entry in the postal industry in Europe.)
} 


$$
p_{1}^{a}=\alpha^{*}=\frac{1+d_{1}-\sqrt{\left(1+d_{1}\right)^{2}-2\left(2 d_{1}+F(1+\beta)\right)}}{2}
$$

Under bypass, the optimal price is given by

$$
p_{1}^{b}=\frac{1-\beta+\beta d_{2}+d_{1}-\sqrt{\left(1-\beta+\beta d_{2}+d_{1}\right)^{2}-4\left(d_{1}\left(1-\beta+\beta d_{2}\right)+F\left(1-\beta^{2}\right)\right)}}{2}
$$

The next graph plots the welfare levels under access and bypass, $W^{a}$ and $W^{b}$ as a function of the delivery cost of the entrant, $d_{2}$ for $\beta=1 / 2, d_{1}=1 / 2$ and $F=1 / 100$.

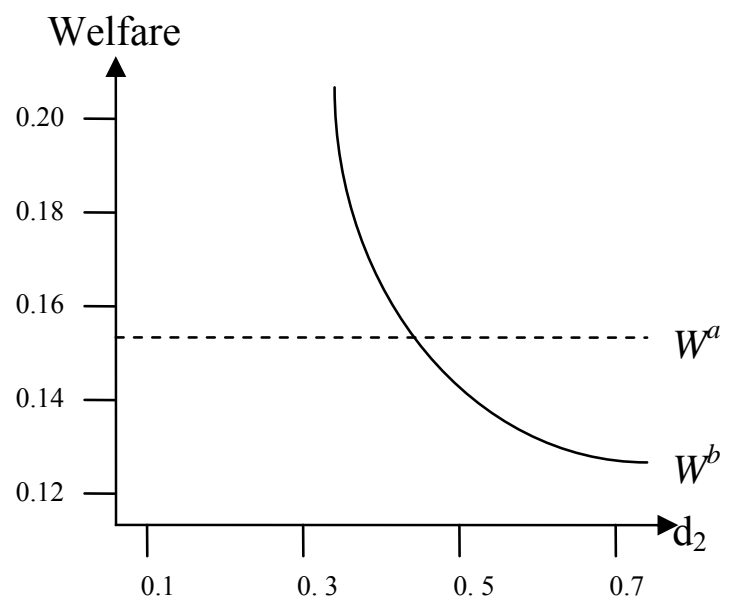

\section{Figure 1: Welfare levels under access and bypass}

The dashed line corresponds to welfare under access. This is independent of the delivery cost of the entrant, and under our parameter values, the incumbent can always cover the fixed cost under access. The solid line corresponds to welfare under bypass. ${ }^{13}$ It is easy to check that if $d_{2}<0.3464$, the incumbent cannot cover his fixed cost, so bypass becomes infeasible. However, bypass is the preferred delivery method for any value $0.3464 \leq d_{2}<0.498$, and access is again preferred for $d_{2} \geq 0.498$.

\subsection{Constrained access}

We now turn to the key issue of the paper. Suppose that the regulator cannot choose the delivery method of the entrant, but is constrained by the decision of the entrant. In that case, firm 2 chooses access whenever $\alpha^{*} \leq d_{2}^{u}$. Consequently, for all the values of $d_{2}^{u} \in\left[d^{*}, \alpha^{*}\right]$, the firm chooses bypass while access is socially preferred. This impact of prices on the delivery method should be incorporated in the design of the optimal prices. In the next subsection, we derive a constrained access solution where the regulator induces the cost-minimizing decision. In the constrained access case, the regulator maximizes the

\footnotetext{
${ }^{13}$ In this simple example, welfare under bypass is monotonically decreasing in $d_{2}$. However, this need not be the case in general.
} 
welfare $W^{a}$ under the additional constraint that $\alpha^{u} \leq d_{2}^{u}$. This constraint aims at inducing an efficient technological choice by firm 2 . When this constraint binds, the efficient stamp prices for the incumbent operator are:

(16) $\frac{p_{1}^{u}-c_{1}-d_{1}^{u}}{p_{1}^{u}}=\frac{\lambda}{1+\lambda} \frac{1}{\hat{\eta}_{1}^{u}}+\left(d_{2}^{u}-d_{1}^{u}\right) \tilde{\gamma}_{1}^{u}$

(17) $\frac{p_{1}^{r}-c_{1}-d_{1}^{r}}{p_{1}^{r}}=\frac{\lambda}{1+\lambda} \frac{1}{\hat{\eta}_{1}^{r}}+\left(d_{2}^{u}-d_{1}^{u}\right) \tilde{\gamma}_{1}^{r}$

with $\tilde{\gamma}_{1}^{u}>0$ and $\tilde{\gamma}_{1}^{r}<0$.

The structure of price under constrained access is similar to the structure under access. The main difference is that the " $\widetilde{\gamma}$ " terms are weighted by the difference in the marginal costs of delivery. Hence, when the delivery cost of the entrant decreases, the urban price decreases while the rural price increases. We now consider under which conditions the regulator chooses access and bypass on the market.

PROPOSITION 2: When the entrant is free to choose whether or not to bypass, the efficient delivery method is access for $d_{1}^{u} \leq d_{2}^{u}$ and bypass for $d_{1}^{u} \geq d_{2}^{u}$.

Proof: (1) For $d_{1}^{u} \leq d_{2}^{u}$, by the same argument as in the proof of Proposition 1, the regulator could choose to set the access price so that $\alpha^{u}=d_{2}^{u}$ and obtain the level of welfare $\bar{W}^{a} \geq W^{b}$. By a revealed preference argument, the regulator always prefers access to bypass.

(2) For $d_{1}^{u} \geq d_{2}^{u}$, we will show that $W^{b} \geq W^{a}$ (with strict inequality if $d_{1}^{u}>d_{2}^{u}$ ), so that the regulator prefers bypass to access. Consider the optimal choice of prices under access, and let $p_{1}^{u b}=p_{1}^{u a}, p_{1}^{r b}=p_{1}^{r a}$. Notice that $p_{2}^{u}=d_{2}^{u}+c_{2}=\alpha^{u}+c_{2}$ both under bypass and access. Let $\bar{W}^{b}$ be the welfare value of bypass under these prices. Because $d_{2}^{u} \leq d_{1}^{u}$, $W^{a} \leq \bar{W}^{b}$. Now, notice that $\bar{\Pi}_{1}^{b}=\Pi_{1}^{a}+\left(d_{1}^{u}-d_{2}^{u}\right) x_{2}^{u} \geq \Pi_{1}^{a}=0$, so these prices are feasible in the case of bypass. Again, by a revealed preference argument, we have: $W^{b} \geq \bar{W}^{b} \geq W^{a}$.

Notice that, if $d_{2}^{u}=d_{1}^{u}$, then $\bar{W}^{a}=W^{b}$. In fact, if $d_{2}^{u}=d_{1}^{u}$, the optimal choices of $p_{1}^{u}$ and $p_{1}^{r}$ are the same under access and bypass, because the two problems faced by the regulator are exactly identical.

This shows that the regulator will always promote bypass if $d_{2}^{u} \leq d_{1}^{u}$ and access if $d_{2}^{u} \geq d_{1}^{u}$. This is the technologically efficient choice, but it is not welfare maximizing. If he could, the regulator would like to favor access for some values such that $d_{2}^{u} \leq d_{1}^{u}$. On the other hand, the regulator will never want to favor bypass for some values such that $d_{2}^{u} \geq d_{1}^{u}$. So there might be excess bypass, but there will never be excess access.

This proposition shows that, if the entrant can freely choose his delivery method, he will base his decision on the comparison between marginal costs of delivery, not taking 
into account the USO of the incumbent. This will result in excess bypass, and in a lower level of welfare than when the regulator can choose the delivery method of the entrant. This welfare loss will not arise when the delivery cost of the entrant is high (namely, when $d_{2}^{u} \geq \alpha^{*}$ ). If $d_{1}^{u} \leq d_{2}^{u}<\alpha^{*}$, the regulator will be forced to lower his access price below the second-best level (from $\alpha^{*}$ to $d_{2}^{u}$ ). If $d^{*} \leq d_{2}^{u}<d_{1}^{u}$, the regulator will have to accept bypass whereas she would have preferred access. Finally, for low values of $d_{2}^{u}$ for which bypass is optimal and feasible, there is no welfare loss, as bypass is always preferred by the regulator. We summarize this discussion with the following figure.

\begin{tabular}{l|l|l|l}
$\begin{array}{l}\text { Bypass optimal } \\
\text { and feasible }\end{array}$ & $\begin{array}{l}\text { Bypass but } \\
\text { access optimal }\end{array}$ & $\begin{array}{l}\text { Access with } \\
\text { suboptimal fee }\end{array}$ & $\begin{array}{l}\text { Access optimal } \\
\text { and feasible }\end{array}$ \\
& $d^{*}$ & $d_{1}^{u}$ & $\alpha^{*}$
\end{tabular}$d_{2}^{u}$

Figure 2: Constrained access and optimal delivery method

EXAMPLE 2: Example 1 continued.

We now compare welfare when the regulator can choose the delivery method and faces constrained access. The following graph shows the value of welfare for the same parameter values as in Example 1. We focus on the region of delivery costs of the entrant where the two welfare levels are different.

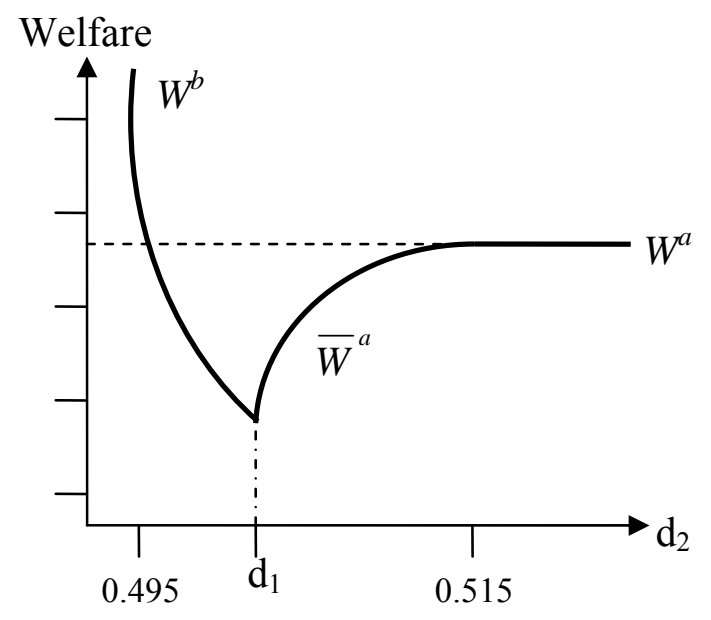

Figure 3: Welfare levels under access, constrained access and bypass

The solid line denotes welfare when the entrant chooses the delivery method and the dashed line welfare under access when the regulator chooses the delivery method. It appears that most of the welfare loss occurs when the two delivery costs are very close. For $0.498 \leq d_{2}^{u} \leq 0.5$, the welfare loss is due to the fact that the regulator faces bypass and prefers access $\left(W^{b} \leq W^{a}\right)$; for $0.5 \leq d_{2}^{u} \leq 0.515$, the welfare loss $\left(\bar{W}^{a} \leq W^{a}\right)$ is due to the fact that the regulator must charge an access price $\alpha=d_{2}^{u}$ which is below the optimal access charge. 
In this section, we have shown that the socially efficient delivery method does not coincide with the technologically efficient one. If the regulator selects the optimal (or Ramsey) prices, ignoring the impact of prices on the delivery choice, there is too much bypass from both a social and technological point of view. Once the regulator takes into account the impact of the access price on the access vs. bypass decision of the entrant, it implements a cost minimizing delivery method but there is still too much bypass with respect to the welfare maximizing outcome.

\section{Extensions}

\subsection{Uniform tariff}

In the postal sector, a geographically uniform tariff is often imposed as part of the universal service obligations. When a unique stamp price $p_{1}$ is applied for urban and rural mails, urban mails are relatively more expensive while rural mails are relatively less expensive: $p_{1}^{u} \leq p_{1} \leq p_{1}^{r}$.

What are the consequences of the uniform tariff on the access vs. bypass decision of the entrant? A higher urban price means that with a given price $p_{2}^{u}$, the entrant is able to capture a larger fraction of the incumbent's customers. As a consequence, the USP has lower receipts from its urban mail. To compensate, the entrant should contribute more to the USO financing. That is, the access price must increase. If we take the first-order condition of the maximization of $W^{a}$, when a uniform tariff is imposed (6) must be replaced by:

(18) $\alpha^{u}=d_{1}^{u}+\frac{\lambda}{1+\lambda} \frac{p_{2}^{u}}{\eta_{2}^{u}}+\underbrace{\left(p_{1}-c_{1}-d_{1}^{u}\right) \sigma^{u}}_{\text {higher displacement term }}$

In this expression, it is clear that the displacement term is higher when a uniform tariff is imposed. And therefore, the optimal access price $\alpha^{*}$ increases when the firm cannot price discriminate between its rural and its urban mail. But, since firm 2 chooses to bypass whenever $d_{2}^{u} \leq \alpha^{*}$, an increase in the access price implies that the set of parameters under which the entrant has incentives to bypass is larger. In other words, this means that if the regulator wants to implement an efficient technological choice by the entrant, the constrained access solution applies for a larger set of parameters under a uniform tariff. This is represented in figure 4. 


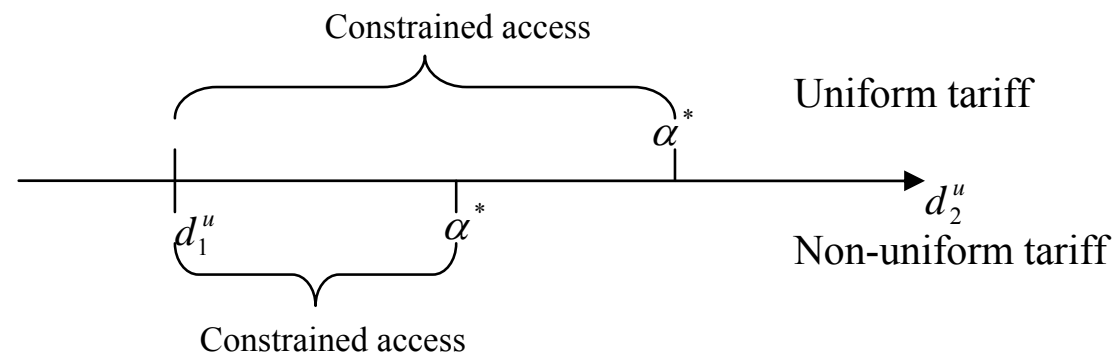

\section{Figure 4: Efficient access vs bypass choice in the uniform and the non-uniform tariff case}

By using the same argument as in proposition 2, we can show that the efficient delivery method is the cost-minimizing one: bypass for $d_{2}^{u}<d_{1}^{u}$ and access otherwise.

The uniform tariff is by itself a source of reduction in the welfare. But when the entry mode is taken as endogenous, constrained access should be applied for a larger set of parameters. Hence there is an additional welfare loss associated with a geographically uniform tariff: whenever the entrant's delivery cost lies in $\left[d_{1}^{u}, \alpha^{*}\right]$, the welfare is $\bar{W}^{a}$ which is lower than the welfare with unconstrained access $W^{a}$.

\subsection{Uncertainty}

We now investigate the consequences of technological uncertainty on the optimal prices and the induced choice of delivery by the entrant. This part of the paper is motivated by the facts that (1) competition in the delivery activity is still at its infancy and (2) the business model of the entrant is different from the incumbent's. Therefore, the regulator may have incomplete information on the entrant's precise delivery cost and we show in this section that this lack of information could be a source of inefficient bypass by the entrant.

More precisely, we consider the following problem: firm 2, the entrant, is characterized by a delivery cost $d_{2}^{u}$, which is private information to the firm. However, the distribution of this cost is common knowledge. In particular, we assume that it is commonly known that $d_{2}^{u}$ is distributed according to a continuous density function $g($.) over the interval $\left[\underline{d}_{2}^{u}, \bar{d}_{2}^{u}\right]$ and $g(\tau)>0$ for all $\tau$ in the interval.

The regulator sets the incumbent's prices ignoring the true value of $d_{2}^{u}$. Firm 2 observes these prices and decides whether it enters the market with its own delivery network or with access. Then, it sets its price at the corresponding competitive level. We concentrate on the cases where the incumbent's prices cannot be contingent on the realized value of $d_{2}^{u} \cdot 14$

The uncertainty on the technology of the entrant translates into an uncertainty on the choice of the delivery method. The key issue in this problem is that the access price $\alpha^{u}$

\footnotetext{
${ }^{14}$ We do not treat the problem as a mechanism design problem where the regulator offers a menu of prices contingent on the revealed value of the delivery cost. If this mechanism is incentive compatible, the firm truthfully reveals its cost. Instead, we assume that the regulator is bound to use flat stamp and access prices. This could be viewed as an application of the non-discrimination principle imposed by the European directives regarding the organization of the postal sector.
} 
determines the probabilities of access and bypass. In particular, for any $\alpha^{u} \in\left[\underline{d}_{2}^{u}, \bar{d}_{2}^{u}\right]$, there is a probability $G\left(\alpha^{u}\right)$ that firm 2 builds up its own delivery network and a probability $1-G\left(\alpha^{u}\right)$ that it uses the incumbent's network, where $G($.$) is the distribution$ function associated with $g():. G(x)=\int_{\underline{d}_{2}^{u}}^{x} g(\tau) d \tau$.

The regulatory problem with unknown cost is then:

$$
\underset{p_{1}^{u}, p_{1}^{r}, \alpha^{u}}{\operatorname{Max}} \hat{\mathrm{W}}=\int_{\underline{\mathrm{d}}_{2}^{u}}^{\alpha^{u}} W^{b} g(\tau) d \tau+\int_{\alpha^{u}}^{\bar{d}_{2}^{u}} W^{a} g(\tau) d \tau
$$

subject to the zero profit constraint for firm 1:

$$
\Pi_{1}=\int_{\underline{\underline{d}}_{2}^{u}}^{\alpha^{u}} \Pi_{1}^{b} g(\tau) d \tau+\int_{\alpha^{u}}^{\bar{d}_{2}^{u}} \Pi_{1}^{a} g(\tau) d \tau \geq 0
$$

$\hat{\mathrm{W}}$ is the expected welfare when the entrant's cost is unknown. It is composed of two terms: the first term is the expected welfare under bypass (the entrant's cost is in $\left[\underline{d}_{2}^{u}, \alpha^{u}\right]$ ); the second term is the welfare under access (the entrant's cost is in $\left[\alpha^{u}, \bar{d}_{2}^{u}\right]$ ). Similarly, the profit of firm 1 is the sum of the expected profit under access and the expected profit under bypass.

We will consider the case in which $d_{1}^{u} \leq \underline{d}_{2}^{u}<\alpha^{*}$. That is the case in which under full information the regulator always prefers access. Moreover, to induce this choice under symmetric information, the regulator must apply the constrained access solution for $d_{2}^{u} \leq \alpha^{*}$.

We now derive a sufficient condition under which $\alpha^{u}=\underline{d}_{2}^{u}$ is not optimal, that is, there exist realizations of the cost for which bypass is chosen. To this end, we denote by $\underline{p}_{1}^{u}$ and $\underline{p}_{1}^{r}$ the price that a regulator would apply if it wants to induce access when it faces an entrant with a known delivery cost of $\underline{d}_{2}^{u}$. Since $\underline{d}_{2}^{u} \leq \alpha^{*}$, these prices are given by equations (16) and (17). We check that $\left\langle\underline{p}_{1}^{u}, \underline{p}_{1}^{r}, \alpha^{u}=\underline{d}_{2}^{u}\right\rangle$ is not a solution to our problem.

In this problem, the regulator faces a trade-off between promoting the efficient delivery technology which requires a low access price and raising funds from the entrant to finance the cost of the USO which requires instead a high access charge. We show that there are circumstances under which the regulator induces "too much" bypass to increase the financial contribution of firm 2 in the case of access. Allowing for inefficient bypass is a way to increase the expected financial contribution of the entrant to the USO. Under asymmetric information, the regulator trades off the contribution of the entrant to the USO financing and the efficient delivery method.

Let us define $\Delta$ as the value of $\frac{\partial\left(W^{a}+\lambda \Pi_{1}^{a}\right)}{\partial \alpha^{u}}$ evaluated at the proposed solution $<\underline{p}_{1}^{u}, \underline{p}_{1}^{r}, \alpha^{u}=\underline{d}_{2}^{u}>$ 
We can show that:

PROPOSITION 3: When, under symmetric information, access is efficient for all possible realizations of $d_{2}^{u}, \underline{d}_{2}^{u} \geq d_{1}^{u}$; there is a positive probability of bypass under asymmetric information $\left(\alpha^{u}>\underline{d}_{2}^{u}\right)$ if: (1) $\underline{d}_{2}^{u}<\alpha^{*}<\bar{d}_{2}^{u}$ and (2) $\Delta \geq x_{2}^{u} g\left(\underline{d}_{2}^{u}\right)\left(\underline{d}_{2}^{u}-d_{1}^{u}\right)$.

\section{Proof: See appendix.}

This proposition demonstrates that whenever access is the socially preferred delivery method for all possible realizations of the cost parameter of the entrant, the regulator may nevertheless select an access price that induces a positive probability of bypass. The reason is that promoting access in all possible circumstances is costly because the regulator must set the access price at the lowest possible level. Then, the regulator allows some bypass to increase the expected access receipts when the firm chooses access. There is a trade-off between an efficient market structure and the USO financing. Uncertainty about the entrant's cost is then a source of excess bypass.

Gautier and Mitra (2008) have a similar result in a context where an entrant with unknown cost decides between access and no entry. They show that allowing for an inefficient market structure ex-post (in their case, a low probability of entry) is a way to increase the financial contribution of the entrant to the infrastructure financing.

If we have a closer look at the condition that guarantees a positive probability of bypass, we can establish that this condition is more likely to be met when the gap between the lowest possible delivery cost of the entrant and the incumbent's marginal delivery cost $\left(\underline{d}_{2}^{u}-d_{1}^{u}\right)$ is small. The reason is that the left hand side $(\Delta)$ decreases in $\left(\underline{d}_{2}^{u}-d_{1}^{u}\right)$ while the right hand side increases in this difference. And, when $\underline{d}_{2}^{u}=d_{1}^{u}$, there is, for sure, a positive probability of bypass.

\section{$5 \quad$ Concluding remarks}

In this paper, we have analyzed the optimal prices chosen by a regulator in the face of entry in the postal sector. We have emphasized that the entrant can choose between two business models: access and bypass, and that the regulator must take this choice into account when computing its optimal prices. If the regulator can control the entrant's choice, he will induce access even when the entrant has a lower delivery cost than the incumbent. If the regulator cannot control the entrant's choice, prices will be chosen so that the entrant prefers to bypass when his delivery cost is lower than the incumbent's, bypass will occur too often, and the USP may be unable to sustain his obligations. The prices that lead to this cost-minimizing outcome are contingent on the delivery cost of the entrant. When the cost of the entrant is unknown, the regulator trades off the probability of bypass with the expected access receipts, and may reduce his access price even more to prevent excessive bypass under uncertainty.

In conclusion, we would like to discuss the welfare implications of the different modes of competition. Competition in the upstream segments of the market (access) is a good thing as long as the entrant offers a differentiated product and/or competition decreases the upstream cost. The case of competing networks (bypass) is more complicated. Bypass raises welfare only when the entrant cost advantage is high enough, but in some 
circumstances, maintaining the USO is not always possible. Preventing bypass with lower access price is not always the solution and it is costly in terms of welfare. Uncertainty leads to excess bypass. And, when access is bought by both competitors and postal customers (usually at the same price), reducing the access price has a more important impact on the receipts of the incumbent.

There are other regulatory options that we did not consider in this paper. First, the scope of the USO could be changed. If $F$ decreases, the margin on all the products decreases and it therefore lowers the incentives to bypass. Reducing the scope of the USO is likely to reduce welfare, but it must be compared with the welfare loss associated with the pricing methods to deter bypass. Second, in the US, the incumbent's monopoly position on the last mail delivery is maintained and it is a radical solution to prevent efficient and inefficient bypass. But, it does not give incentives to the incumbent for adopting a more efficient delivery technology unless it has the option to sub-contract delivery (see Panzar, 2005 for a detailed analysis of competition for delivery). Finally, Armstrong (2001) proposes to set up a compensation fund by selling access at marginal cost and applying an output tax $t$ to the competitor, irrespective of its delivery method. This is a way to achieve the cost-minimizing outcome and to attain welfare level $W^{a}$ if $t=\alpha^{*}-d_{1}^{u}$ under access. However, an output tax and a compensation fund for the USO are not easily implemented in the postal sector.

\section{$6 \quad$ References}

Armstrong, M. (2001) “Access Pricing, Bypass and Universal Service," American Economic Review, Papers and Proceedings, 91: 297-301.

Armstrong, M., C. Doyle and J. Vickers (1996) "The Access Pricing Problem: A Synthesis," Journal of Industrial Economics, 44: 131-150.

Baumol, W., J. Panzar and R. Willig (1982) Contestable Markets and the Theory of Industry Structure. Harcourt, Brace, Jovanovich: New York.

Billette de Villemeur, E., H. Cremer, B. Roy. and J. Toledano (2007) "Worksharing, Access and Bypass: the Structure of Prices in the Postal Sector," Journal of Regulatory Economics, 32: 67-85.

Cazals, C., M. de Rycke, J.P. Florens and S. Rouzaud (1997) "Scales Economies and Natural Monopolies in the Postal Delivery," in Crew and P. Kleindorfer (ed.), Managing Changes in the Postal and Delivery Industries, Kluwer Academic Press, Boston.

Cremer, H., M. De Rycke and A. Grimaud (1997) "Costs and Benefits of Universal Service Obligations in the Postal Sector," in Crew and P. Kleindorfer (ed.), Managing Changes in the Postal and Delivery Industries, Kluwer Academic Press: Boston.

Crew, M. and P. Kleindorfer (1992) The Economics of Postal Services. Kluwer Academic Publishers: Boston. 
Crew, M. and P. Kleindorfer (2005) "Competition, Universal Service and the Graveyard Spiral," in M. Crew and P. Kleindorfer (ed.), Regulatory and Economics Changes in the Postal and Delivery Sector, Kluwer Academic Publishers, Boston.

Crew, M. and P. Kleindorfer (2006) "Welfare Effects of Entry and Strategies for Maintaining the USO," in M. Crew and P. Kleindorfer (ed.), Progress Toward the Liberalization of the Postal and Delivery Sector, Springer, New York.

Dam, K., A. Gautier, and M. Mitra (2007) "Efficient Access Pricing and Endogenous Market Structure,” CORE DP 2007/4, Louvain-la-Neuve, Belgium.

De Donder, P. (2006) "Access Pricing in the Postal Sector: Theory and Simulations," Review of Industrial Organization, 28: 307-326.

De Donder, P., H. Cremer, P. Dudley, and F. Rodriguez (2006) "Pricing and Welfare Implications of Alternative Approaches to Setting Price Controls in the Postal Sector," in M. Crew and P. Kleindorfer (ed.), Progress Toward the Liberalization of the Postal and Delivery Sector, Springer, New York.

Gautier, A. and M. Mitra (2008) "Regulation of an Open Access Essential Facility," Economica, forthcoming.

Laffont, J. J. and J. Tirole (1994) "Access Pricing and Competition," European Economic Review, 38: 1673-1710.

Laffont, J. J. and J. Tirole (2000) Competition in Telecommunications. MIT Press: Cambridge and London.

Panzar, J. (2005) "Combining Liberalization and Unbundling Policies in Postal Markets," paper presented at the 13th CRRI conference on Postal and Delivery Economics.

Price Waterhouse Coopers (2006) The Impact on Universal Service of the Full Market Accomplishment of the Postal Internal Market in 2009, report to the European Commission.

Rohlfs, J. (1979) "Economically-efficient Bell System Pricing," Bell Labs Discussion Paper 138.

\section{$7 \quad$ Appendix}

\subsection{Derivation of the optimal prices}

Let us define the following $2 \times 2$ matrix $A$ and the column vector $b$ : 


$$
A=\left(\begin{array}{cc}
-x_{1}^{u} \eta_{1}^{u} & x_{1}^{r} \frac{p_{1}^{r}}{p_{1}^{u}} \eta_{1}^{r u} \\
x_{1}^{u} \frac{p_{1}^{u}}{p_{1}^{r}} \eta_{1}^{u r} & -x_{1}^{r} \eta_{1}^{r}
\end{array}\right), b=\left(\begin{array}{c}
-\frac{\lambda}{1+\lambda} x_{1}^{u} \\
-\frac{\lambda}{1+\lambda} x_{1}^{r}
\end{array}\right)
$$

From these matrices, we define the $2 \times 2$ matrix $B_{i}$ as the matrix $A$ where its $i^{\text {th }}$ column is replaced by the vector $b$.

The solutions of the equation system

$$
A\left(\begin{array}{l}
X_{1} \\
X_{2}
\end{array}\right)=b
$$

are:

$$
\begin{aligned}
& X_{1}=\frac{\operatorname{det}\left|B_{1}\right|}{\operatorname{det}|A|}=\frac{\lambda}{1+\lambda} \frac{\eta_{1}^{r}+\eta_{1}^{r u}}{\eta_{1}^{r} \eta_{1}^{u}-\eta_{1}^{r u} \eta_{1}^{u r}}=\frac{\lambda}{1+\lambda} \frac{1}{\hat{\eta}_{1}^{u}} \\
& X_{2}=\frac{\operatorname{det}\left|B_{2}\right|}{\operatorname{det}|A|}=\frac{\lambda}{1+\lambda} \frac{\eta_{1}^{r}+\eta_{1}^{u r}}{\eta_{1}^{r} \eta_{1}^{u}-\eta_{1}^{r u} \eta_{1}^{u r}}=\frac{\lambda}{1+\lambda} \frac{1}{\hat{\eta}_{1}^{r}}
\end{aligned}
$$

To derive these expressions, we made the hypothesis that $d x_{1}^{u} / d p_{1}^{r}=d x_{1}^{r} / d p_{1}^{u}$.

\subsubsection{Access}

Under access, the first-order conditions of the welfare maximization problem can be expressed as:

$$
\left(\begin{array}{ccc}
-x_{1}^{u} \eta_{1}^{u} & x_{1}^{r} \frac{p_{1}^{r}}{p_{1}^{u}} \eta_{1}^{r u} & x_{2}^{u} \frac{p_{2}^{u}}{p_{1}^{u}} \eta_{2}^{u u} \\
x_{1}^{u} \frac{p_{1}^{u}}{p_{1}^{r}} \eta_{1}^{u r} & -x_{1}^{r} \eta_{1}^{r} & 0 \\
x_{1}^{u} \frac{p_{1}^{u}}{p_{2}^{u}} \eta_{1}^{u u} & 0 & -x_{2}^{u} \eta_{2}^{u}
\end{array}\right)\left(\begin{array}{l}
M_{1}^{u} \\
M_{1}^{r} \\
M_{2}^{u}
\end{array}\right)=\left(\begin{array}{c}
-\frac{\lambda}{1+\lambda} x_{1}^{u} \\
-\frac{\lambda}{1+\lambda} x_{1}^{r} \\
-\frac{\lambda}{1+\lambda} x_{2}^{u}
\end{array}\right)
$$

The solution is:

$$
M_{1}^{u}=\frac{\lambda}{1+\lambda}\left(\frac{1}{\hat{\eta}_{1}^{u}}+\gamma_{1}^{u}\right)
$$

where $\gamma_{1}^{u}=x_{1}^{r} \eta_{1}^{r} \eta_{2}^{u u} \frac{p_{2}^{u}}{p_{1}^{u}} \frac{x_{2}^{u}+\eta_{1}^{u u} \frac{p_{1}^{u}}{p_{2}^{u}} \frac{1}{\eta_{1}^{u}}}{\eta_{2}^{u} \operatorname{det}|A|-\eta_{2}^{u u} \eta_{1}^{u u} x_{1}^{u} x_{1}^{r} \eta_{1}^{r}}>0$.

And:

$$
M_{1}^{r}=\frac{\lambda}{1+\lambda}\left(\frac{1}{\hat{\eta}_{1}^{r}}+\gamma_{1}^{r}\right)
$$


where $\gamma_{1}^{r}=x_{1}^{u} \eta_{2}^{u u} \frac{x_{2}^{u} \eta_{1}^{u r} \frac{p_{2}^{u}}{p_{1}^{u}}+x_{1}^{r} \eta_{1}^{r}\left(\frac{\eta_{1}^{r}}{\hat{\eta}_{1}^{r}}-1\right)}{\eta_{2}^{u} \operatorname{det}|A|-\eta_{2}^{u u} \eta_{1}^{u u} x_{1}^{u} x_{1}^{r} \eta_{1}^{r}} \cdot \gamma_{1}^{r}<0$ because $\eta_{1}^{u r}<0$ and $\frac{\eta_{1}^{r}}{\hat{\eta}_{1}^{r}}<1$.

\subsubsection{Bypass}

Under bypass, the first-order conditions of the welfare maximization problem can be expressed as:

$$
A\left(\begin{array}{l}
M_{1}^{u} \\
M_{1}^{r}
\end{array}\right)=b
$$

We immediately have: $M_{1}^{u}=\frac{\operatorname{det}\left|B_{1}\right|}{\operatorname{det}|A|}=\frac{\lambda}{1+\lambda} \frac{1}{\hat{\eta}_{1}^{u}}$ and $M_{1}^{r}=\frac{\operatorname{det}\left|B_{2}\right|}{\operatorname{det}|A|}=\frac{\lambda}{1+\lambda} \frac{1}{\hat{\eta}_{1}^{r}}$. Firm 2 sets its price at marginal cost: $p_{2}^{u}=c_{2}+d_{2}^{u}$ and the access price is set at a level that induces bypass: $\alpha^{u} \geq d_{2}^{u}$.

\subsubsection{Constrained access}

Under constrained access, we have $\alpha^{u}=d_{2}^{u}$. The first-order conditions of the welfare maximization problem can be expressed as:

$$
A\left(\begin{array}{l}
M_{1}^{u} \\
M_{1}^{r}
\end{array}\right)=b+\left(\begin{array}{c}
-\left(d_{2}^{u}-d_{1}^{u}\right) \frac{d x_{2}^{u}}{d p_{1}^{u}} \\
0
\end{array}\right)
$$

The solution is:

$$
\begin{gathered}
M_{1}^{u}=\frac{\operatorname{det}\left|B_{1}\right|}{\operatorname{det}|A|}+\left(d_{2}^{u}-d_{1}^{u}\right) \frac{x_{1}^{r} \eta_{1}^{r}\left(d x_{2}^{u} / d p_{1}^{u}\right)}{\operatorname{det}|A|} \\
=\frac{\lambda}{1+\lambda} \frac{1}{\hat{\eta}_{1}^{u}}+\left(d_{2}^{u}-d_{1}^{u}\right) \frac{\underbrace{\frac{\eta_{1}^{r}\left(d x_{2}^{u} / d p_{1}^{u}\right)}{x_{1}^{u}\left(\eta_{1}^{u} \eta_{1}^{r}-\eta_{1}^{r u} \eta_{1}^{u r}\right)}}_{+}}{M_{1}^{r}=\frac{\operatorname{det}\left|B_{2}\right|}{\operatorname{det}|A|}+\left(d_{2}^{u}-d_{1}^{u}\right) \frac{\left(p_{1}^{u} / p_{1}^{r}\right) x_{1}^{u} \eta_{1}^{u r}\left(d x_{2}^{u} / d p_{1}^{u}\right)}{\operatorname{det}|A|}} \\
=\frac{\lambda}{1+\lambda} \frac{1}{\hat{\eta}_{1}^{r}}+\left(d_{2}^{u}-d_{1}^{u}\right) \underbrace{\frac{\eta_{1}^{r u}\left(d x_{2}^{u} / d p_{1}^{u}\right)}{x_{1}^{r}\left(\eta_{1}^{u} \eta_{1}^{r}-\eta_{1}^{r u} \eta_{1}^{u r}\right)}}_{-}
\end{gathered}
$$




\subsection{Proof of proposition 3}

Integrating by parts, the regulator's objective function can be expressed as:

$$
\underset{p_{1}^{u}, p_{1}^{r}, \alpha^{u}}{\operatorname{Wa}} \hat{\mathrm{W}} \equiv W^{a}+\lambda \Pi_{1}^{a}-(1+\lambda) G\left(\alpha^{u}\right)\left(\alpha^{u}-d_{1}^{u}\right) x_{2}^{u}-\Gamma
$$

where $\Gamma=\int_{\underline{d}_{2}^{u}}^{\alpha^{u}} G(\tau)\left(\frac{\partial W^{b}}{\partial d_{2}^{u}}+\lambda \frac{\partial \Pi_{1}^{b}}{\partial d_{2}^{u}}\right) d \tau$.

This objective function is the sum of three terms. The first one is equivalent to the objective function of the regulator under access. It is what the regulator can achieve when it does not allow bypass $\left(\alpha^{u}=\underline{d}_{2}^{u}\right)$. The other two terms measure the impact of allowing bypass with a positive probability. The second term is the expected loss of access receipts. This has an impact on both the welfare and the profit and it therefore explains why it is multiplied by $(1+\lambda) .{ }^{15}$ The third term $\Gamma$ captures the impact on the welfare and the profit of having a more efficient entrant that is, an entrant with a lower delivery cost $d_{2}^{u}$. The entrant's delivery cost does not affect the welfare and the profit under access since $p_{2}^{u}=\alpha^{u}+c_{2}$, but it affects them under bypass since $p_{2}^{u}=d_{2}^{u}+c_{2} . \Gamma$ then measures the impact of a change in $d_{2}^{u}$ and therefore of a change in $p_{2}^{u}$ on the objective function.

The first-order conditions of the above problem are:

$$
\frac{\partial \hat{W}}{\partial p_{1}^{u}}=\frac{\partial\left(W^{a}+\lambda \Pi_{1}^{a}\right)}{\partial p_{1}^{u}}-(1+\lambda) G\left(\alpha^{u}\right)\left(\alpha^{u}-d_{1}^{u}\right) \frac{\partial x_{2}^{u}}{\partial p_{1}^{u}}-\frac{\partial \Gamma}{\partial p_{1}^{u}}=0
$$

(20) $\frac{\partial \hat{W}}{\partial p_{1}^{r}}=\frac{\partial\left(W^{a}+\lambda \Pi_{1}^{a}\right)}{\partial p_{1}^{r}}-\frac{\partial \Gamma}{\partial p_{1}^{r}}=0$

$$
\begin{aligned}
\frac{\partial \hat{W}}{\partial \alpha^{u}} & =\frac{\partial\left(W^{a}+\lambda \Pi_{1}^{a}\right)}{\partial \alpha^{u}}-(1+\lambda) G\left(\alpha^{u}\right)\left(\alpha^{u}-d_{1}^{u}\right) \frac{\partial x_{2}^{u}}{\partial \alpha^{u}} \\
& -x_{2}^{u}\left(G\left(\alpha^{u}\right)+g\left(\alpha^{u}\right)\left(\alpha^{u}-d_{1}^{u}\right)\right)-\frac{\partial \Gamma}{\partial \alpha^{u}}=0
\end{aligned}
$$

\footnotetext{
${ }^{15}$ By integrating by parts, the expected delivery cost of the entrant evaluated at the upper bound of the integral $\left(d_{2}^{u}=\alpha^{u}\right)$ is $G\left(\alpha^{u}\right) \alpha^{u}$. In order to isolate in the objective function the expression $W^{a}$, we express this expected delivery cost as the sum of the expected cost under access $G\left(\alpha^{u}\right) d_{1}^{u}$ plus $G\left(\alpha^{u}\right)\left(\alpha^{u}-d_{1}^{u}\right)$.
} 
with the derivatives of $W^{a}+\lambda \Pi_{1}^{a}$ given by (3), (4) and (5).

We now derive a condition under which $<\underline{p}_{1}^{u}, \underline{p}_{1}^{r}, \alpha^{u}=\underline{d}_{2}^{u}>$ is not a solution to the above problem. If $\alpha^{u}=\underline{d}_{2}^{u}$, we have $G\left(\alpha^{u}\right)=0$ and then, for $k=u, r: \frac{\partial \Gamma}{\partial p_{1}^{k}}=0$ and $\frac{\partial \Gamma}{\partial \alpha}=0$. Then, by construction (19) and (20) are both equal to zero. Then, if evaluated at the proposed solution, (21) is positive, the regulator increases the welfare by increasing $\alpha^{u}$ and the proposed solution is not valid. Because $\underline{d}_{2}^{u}$ is smaller than $\alpha^{*}$, we have that evaluated at the proposed solution $\Delta=\frac{\partial\left(W^{a}+\lambda \Pi_{1}^{a}\right)}{\partial \alpha^{u}}>0$. Then, a sufficient condition for having $\alpha^{u} \geq \underline{d}_{2}^{u}$ is:

$$
\Delta \geq x_{2}^{u} g\left(\underline{d}_{2}^{u}\right)\left(\underline{d}_{2}^{u}-d_{1}^{u}\right) .
$$

